\title{
Body Scanning App: Data-Driven 3D Reconstruction and Applications
}

\author{
Alfredo BALLESTER*, Eduardo PARRILLA, Julio A. VIVAS, Ana PIEROLA, Jordi URIEL, \\ Sergio A. PUIGCERVER, Paola PIQUERAS, Francisco FOS, Marisol RODRIGUEZ, \\ Juan C. GONZALEZ, Sandra ALEMANY \\ Instituto de Biomecánica de Valencia, Universidad Politécnica de Valencia, Valencia, Spain
}

DOI: $10.15221 / 15.184$ http://dx.doi.org/10.15221/15.184

Keywords: 3D, foot, body, scanning, shape, measurement, data-driven, PCA, e-commerce, personalization, size allocation, configuration, virtual try-on, retail, clothing, footwear, orthotics, transports, ergonomics, low-cost, smartphone, app

\begin{abstract}
The access to the 3D representation of people's body shape has multiple applications to consumer goods which performance is related to human body dimensions or shape. This is the case of wearables such as clothing, footwear, headgear, orthotics, or equipment/environments such as furniture, transports or workstations. Some of the existing and potential applications of 3D human representations include personalisation, virtual try-on or size allocation for wearables or product configuration/adjustment for equipment/environments.
\end{abstract}

However, the cost of 3D scanners is high; the devices are too bulky for homes and retail stores; and its proper use requires expertise to get the relevant parameters from the 3D object (e.g. measurements). These three barriers are currently hindering the massive spreading of 3D scanners as consumer good or as typical in-store appliance.

This paper describes an array of approaches for realistically estimating human 3D shapes (i.e. full bodies or feet) using a regular smartphone or just entering a set of parameters (e.g. age, gender and self-taken measurements). The proposed approaches are based on data-driven 3D reconstructions, using parameterised shape spaces created from large 3D human body or feet databases. The algorithm finds the combination of shape parameters that best matches either the silhouettes extracted from the images or the body measurements entered.

Despite not being actual body scanners, these solutions are easy-to-use and can provide enough accuracy for applications such as virtual try-on, made-to-measure or size allocation of certain types of wearables. Moreover, they can be distributed to the final consumer or to the points of sale at a really reduced cost (or even for free), thus overcoming the main barriers to the massive spreading of its use in e-commerce, new retail experiences, new production pipelines or new business models.

In order to illustrate these technologies, some examples of application to different contexts (i.e. virtual worlds, e-commerce and personalisation) are presented: virtual try-on of female fashion (VisuaLook), size allocation for childrenswear (KIDSIZE), personalised comfort insoles (Sunfeet) and personalised shoes at the point of sale (InstantShoe).

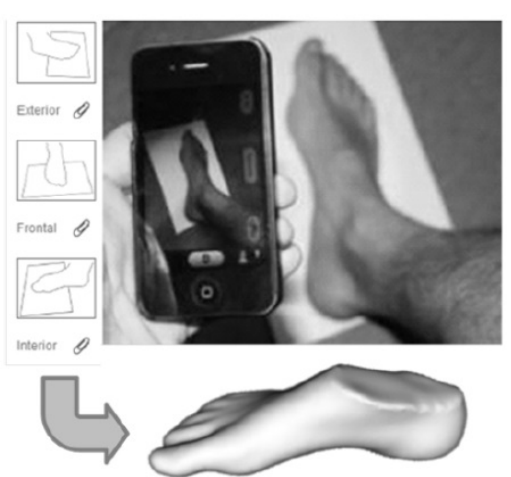

Fig. 1: Foot shape acquisition with a smartphone

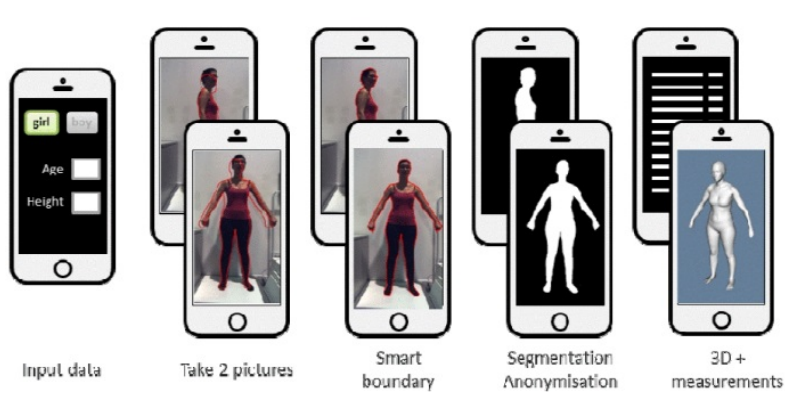

Fig. 2: Full body shape acquisition with a smartphone

* corresponding author alfredo.ballester@ibv.upv.es 